\title{
Chemotherapy-Induced Neuronal Maturation in Sinonasal Teratocarcinosarcoma-a Unique Observation
}

\author{
Shubhada V. Kane $\cdot$ Arti A. Karpate \\ Munita Bal · S. L. Juvekar · Prathmesh S. Pai
}

Received: 7 September 2008/Accepted: 12 November 2008/Published online: 4 December 2008

(C) The Author(s) 2008. This article is published with open access at Springerlink.com

\begin{abstract}
Sinonasal teratocarcinosarcoma (SNTCS) is a rare and highly malignant tumour with combined features of a teratoma and carcinosarcoma. We report the first case of a SNTCS in 23 year old male treated with neo-adjuvant chemotherapy followed by cranio-facial resection. The resection specimen displayed cellular maturation in the neuroectodermal component. The patient presented with a short history of nasal obstruction, epistaxis and headache. On imaging, a bone destroying lesion of left paranasal sinuses and nasal cavity was identified. The diagnosis of SNTCS could be offered only on the third biopsy which showed heterogeneous admixture of primitive neuroectodermal, epithelial and mesenchymal elements. An adequate sampling with high index of suspicion is needed to catch hold this rare tumor. Tumor was excised after 4 cycles of neo-adjuvant chemotherapy. On microscopic examination, it showed similar epithelial and mesenchymal components as the pretreatment biopsies. However, the primitive neuroectodermal component displayed extensive neuronal maturation. The undifferentiated neuroectodermal cells were completely absent in the post chemotherapy specimen. This case throws light on the morphologic evidence of chemotherapy induced maturation in the neuroectodermal component within SNTCS, an event hitherto not reported in the literature in case of SNTCS.
\end{abstract}

Keywords Teratocarcinosarcoma - Sinonasal tumours . Neuronal maturation $\cdot$ Neo-adjuvant chemotherapy

S. V. Kane $(\bowtie) \cdot$ A. A. Karpate · M. Bal ·

S. L. Juvekar · P. S. Pai

Tata Memorial Hospital, Parel, Mumbai, Maharashtra, India

e-mail: drsvkane@gmail.com

\section{Introduction}

Sinonasal teratocarcinosarcoma (SNTCS) is an extremely rare and a highly malignant tumour with combined features of a teratoma and carcinosarcoma. First described as teratoid carcinosarcoma by Shanmugaratnam et al. [1], this enigmatic entity was given its present name of teratocarcinosarcoma by Heffner et al. [2]. Unique in histologic appearance, SNTCS is characterized by tissue heterogeneity, varying maturity with a variegated histologic architecture comprising a complex interplay of neuroectodermal elements, epithelial and mesenchymal proliferations. This histologic heterogeneity, a morphologic hallmark of SNTCS also accounts for numerous diagnostic errors in small biopsies.

SNTCS are highly malignant neoplasms treated with surgery, with or without adjuvant therapies. Histologic maturation of the undifferentiated components of the teratocarcinosarcoma following chemotherapy has never been reported, as the neo-adjuvant chemotherapy is never given in SNTCS (Table 1).

\section{Case Report}

A 23-year old Indian male presented to Tata Memorial Hospital with a 3-month history of nasal stuffiness, nasal bleeding, nasal twang in voice, anosmia and increasing headache. His medical and social history was unremarkable. On examination, he had fullness of the cheek and nose on the left side. Lymphnodes, thyroid and salivary glands were non-palpable. Tumour markers (serum alfafeto protein and beta-human chorionic gonadotropin) were within normal limits. Radiographs and computerizedtomography scans showed a large bone destroying and 
Table 1 Antibodies used for tumour cell characterization

\begin{tabular}{lll}
\hline IHC & Company & Dilution \\
\hline MIC2 & Dako & $1: 100$ \\
NSE & Dako & $1: 100$ \\
Synaptophysin & Dako & $1: 100$ \\
Chromogranin & Dako & $1: 100$ \\
GFAP & Dako & $1: 1200$ \\
S100 & Dako & $1: 600$ \\
NFP & Dako & $1: 100$ \\
CK-7 & Dako & $1: 100$ \\
HMWCK & Dako & $1: 100$ \\
EMA & Dako & $1: 200$ \\
P63 & Dako & $1: 200$ \\
Vimentin & Dako & $1: 200$ \\
SMA & Dako & $1: 400$ \\
\hline
\end{tabular}

heterogeneously enhancing lobulated mass involving the left nasal cavity, maxillary, ethmoid and frontal sinuses, right nasal cavity with intracranial and retrobulbar extension (Fig. 1a). His blood counts and biochemical investigations were within normal limits.

A course of neoadjuvant chemotherapy comprising four cycles of cisplastin and adriamycin was administered to the patient. The post-chemotherapy MRI scan revealed a partial response in the extracranial portion, with no response in the intracranial part (Fig. 1b). Subsequently, an endoscopic-assisted craniofacial resection was performed on the patient for a T3N0 sinonasal tumour.

The first biopsy submitted revealed crushing artifacts, predominantly large necrotic areas with few degenerating atypical round cells within. Limited and degenerating tumour tissue precluded an immunohistochemical evaluation, and differential diagnoses of lymphoma, olfactory neuroblastoma (ONB) and alveolar rhabdomyosarcoma were suggested in this young patient. The second biopsy revealed only malignant squamous epithelial component showing basaloid features with fibrous stroma without even trace of round cells leading to the diagnosis of squamous cell carcinoma. Review of the histopathologic features of these two biopsies raised a suspicion of a SNTCS and a generous biopsy was requested. The third biopsy, submitted as multiple fragments, showed a heterogeneous malignant tumour composed of three different elements in a necrotic background (Fig. 2). Most preponderant was a malignant round cell component, immunopositive for mic2 and NSE (neuron-specific enolase) representing primitive neuroectodermal tissue occupying nearly $40 \%$ of the tumour volume in the biopsy. This component was intimately admixed with an epithelial component composed of malignant squamous epithelium and benign glands lined by cuboidal epithelium, embedded in mesenchymal tissue composed of myofibroblasts. No ganglion cells or neurofibrillary matrix was identified. On the basis of the specific histomorphology, as seen in three biopsies, a confident diagnosis of SNTCS was rendered.

Patient underwent a craniofacial resection for a T3N0 sinonasal tumour following the neo-adjuvant chemotherapy. The specimen was received in multiple, partly hemorrhagic soft-to-rubbery tan-coloured tissue bits, having variegated appearance, the largest one measuring $2.5 \times 2.0 \times 1.0 \mathrm{~cm}$. The entire tissue was submitted for processing. On microscopy, the tumour was heterogeneous and composed of intimate admixture of neuroectodermal, epithelial and mesenchymal components (Fig. 3). The neuroectodermal component was composed of immature ganglions cells embedded in abundant neurofibrillary matrix (Fig. 4). Although all the 16 hematoxylin and eosinstained sections exhibited varying proportions of neurofibrillary matrix, it was predominant in the intracranial portion of the tumour. None of the sections showed primitive neuroectodermal cells as seen in the prechemotherapy biopsy. Embedded within the neurofibrillary
Fig. 1 a A large enhancing mass in the nasoethmoidal region with erosion of cribriform plate and large intracranial extension in the anterior cranial fossa. (prechemotherapy MRI). b Decrease in the size of the mass as compared to the prechemotherapy MRI (Postchemotherapy MRI)
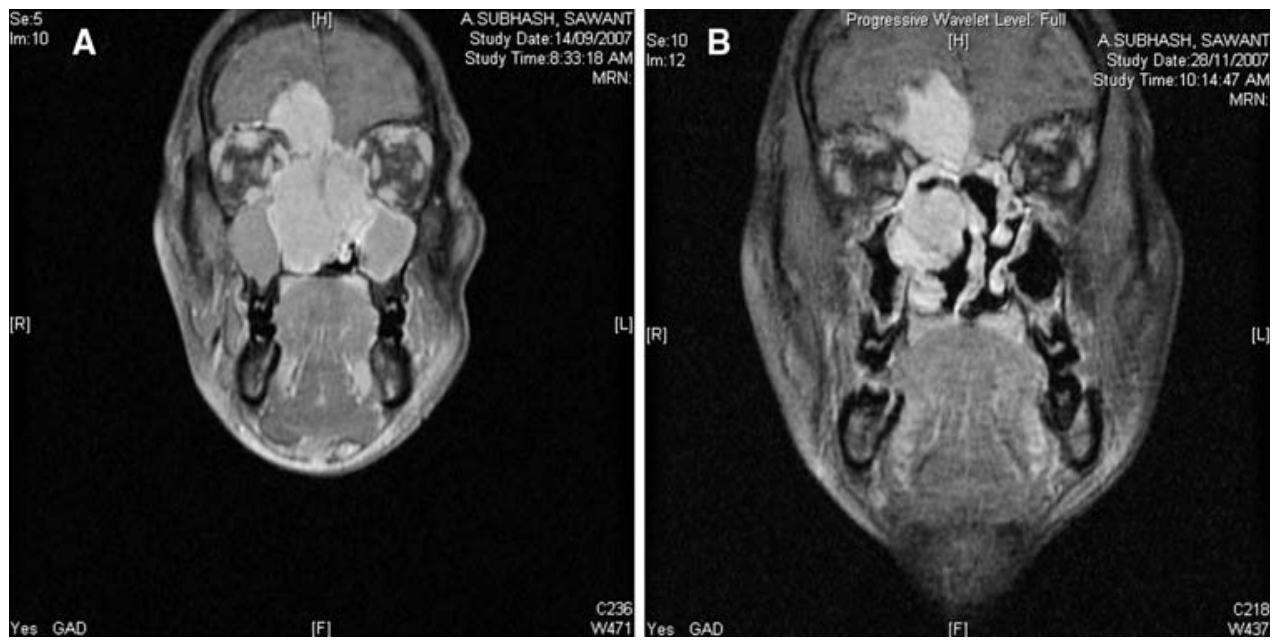
Fig. 2 a Admixture of the primitive neuroectodermal \& benign mesenchymal component, as seen in the biopsy (200× Hematoxylin \& Eosin). b Epithelial component in the form of a dilated gland with transition into squamous epithelium surrounded by benign mesenchymal component $(400 \times$ Hematoxylin \& Eosin)

Fig. 3 a \& b Various components of the tumour in the form of heterogeneous admixture of glandular structures, nodules of neural tissue with neurofibrillary matrix and fibroblastic stroma, as seen in the resection specimen. (A-100×, B-200× Hematoxylin \& Eosin)
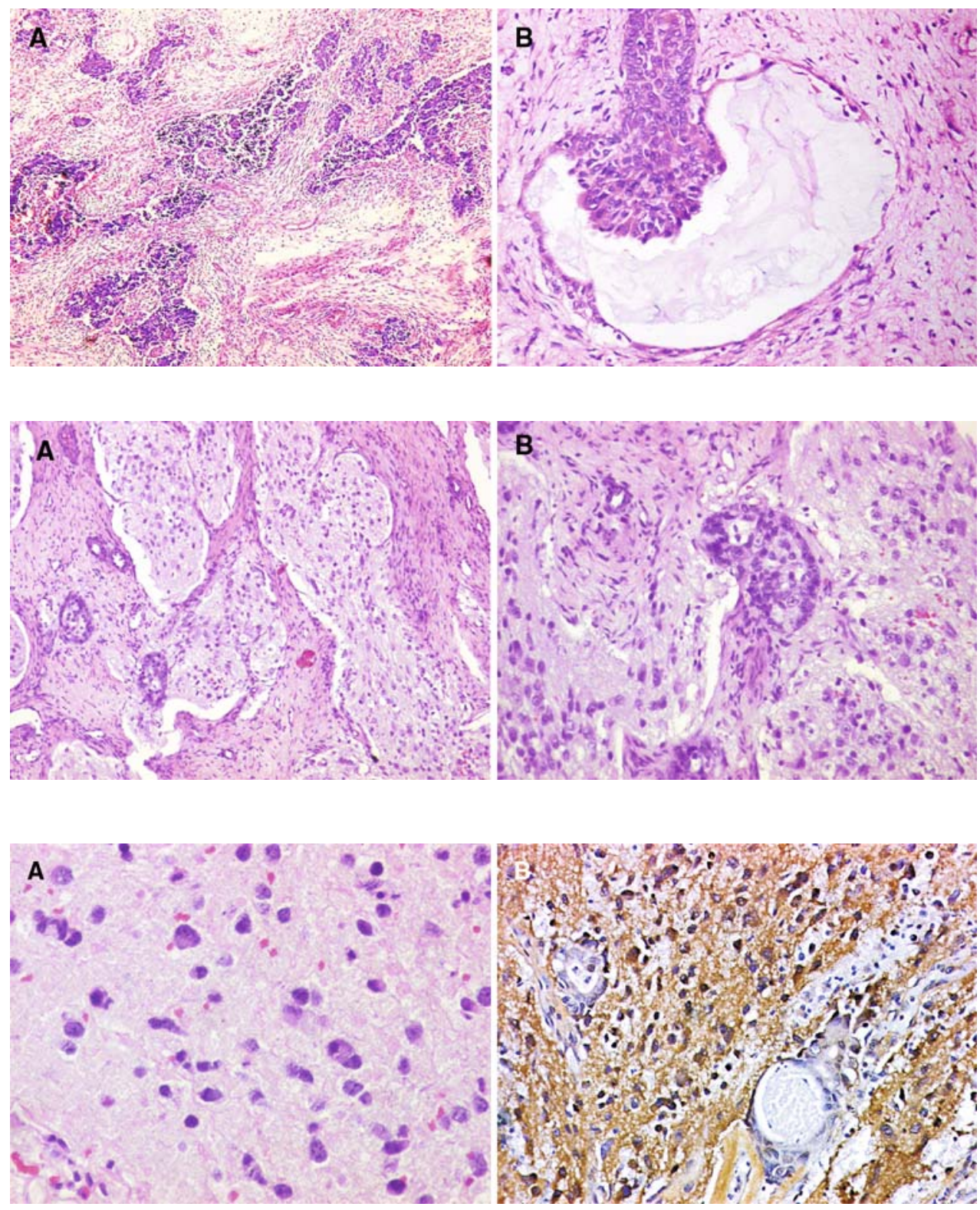

Fig. 4 a Ganglion-like neuronal cells embedded in abundant mature neurofibrillary matrix (Hematoxylin \& Eosin $400 \times$ ). b Synaptophysin reactivity in neurofibrillary matrix $(400 \times)$ matrix, numerous invasive epithelial islands composed of malignant squamous elements and characteristic hybrid squamo-glandular units were noted. A moderate atypia and mitotic activity were demonstrated in the epithelial elements. Mesenchymal component was composed of myofibroblastic proliferation with collagenized stroma. No obvious sarcomatous component was identified. Bone or cartilage was not seen. Surgical margins were positive for tumour. Scanty uninvolved brain paranchyma was also noted. Mature neuronal component was immunoreactive to neurofilament protein (NFP) [1:100; DAKO] (Fig. 5), NSE [1:100; Dako], synaptophysin [1:100; Dako], chromogranin [1:100; DAKO] and GFAP [1:1200; DAKO]. S-100 protein was focally positive. It was immunonegative for MIC2 [1:100; DAKO]. The glandular epithelial component was immunoreactive to epithelial membrane antigen
[1:200; DAKO] and CK7 [1:100; DAKO] while the squamous component was positive for high molecular weight cytokeratin [1:100; DAKO] and p63 [1:200; DAKO] (Fig. 6). Positive labelling with vimentin and smooth muscle actin [1:400; DAKO] was observed in the mesenchymal component. There was no evidence of a germinoma, embryonal carcinoma, yolk-sac tumour or choriocarcinoma in any of the sections.

The patient had received radiotherapy postoperatively and is free of disease 10 months post surgery.

\section{Discussion}

SNTCS is an extremely rare and highly malignant tumour with a unique and complex histology. Very few articles 
Fig. 5 a NFP positivity in the nodules of immature neural tissue with neurofibrillary matrix. b Note the immunonegativity in the mature epithelial component seen in the centre

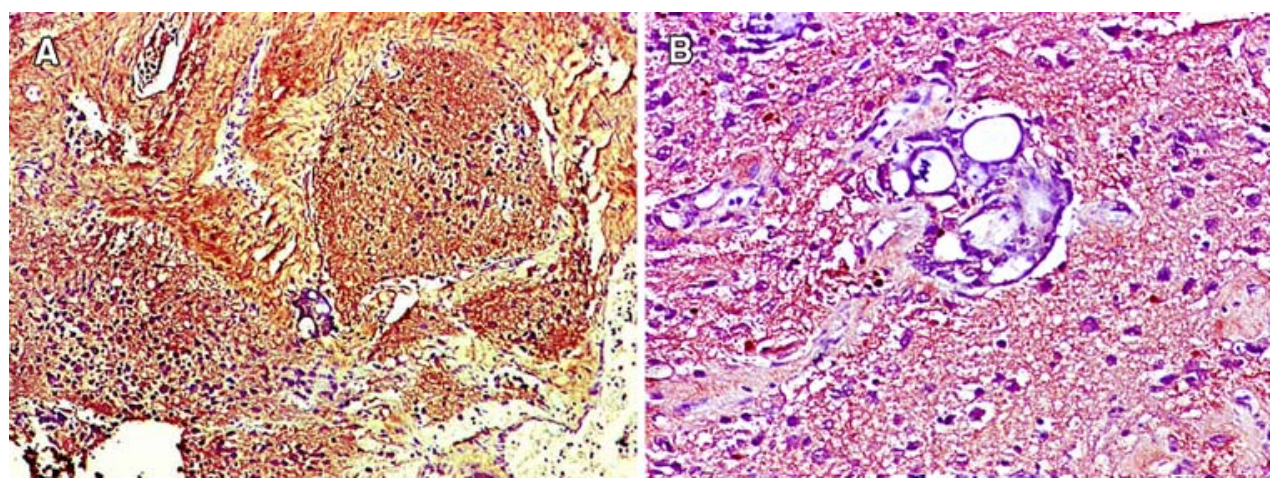

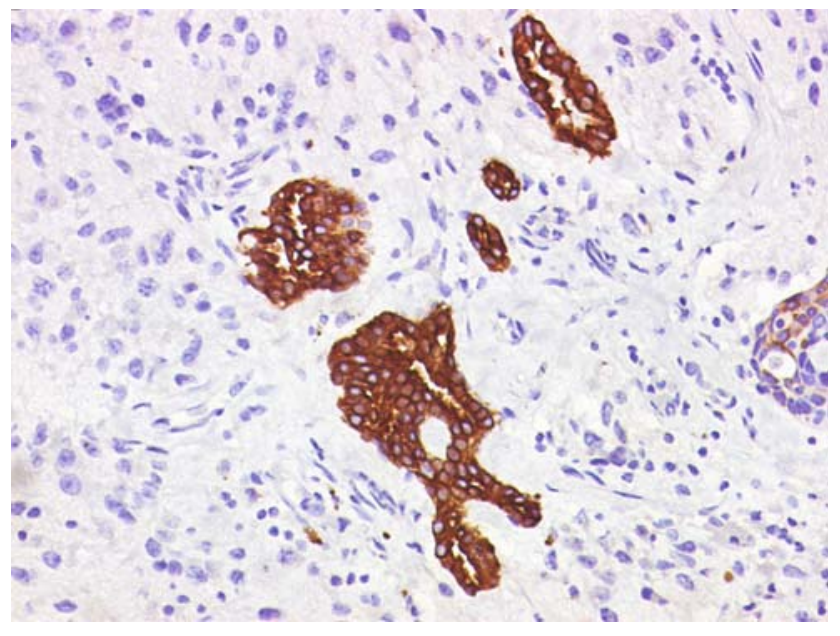

Fig. 6 CK-7 immunoreactivity in the epithelial component $(200 \times)$

mainly in the form of case reports and small series of cases comprising not more than 20 cases are available in the literature [2]. First described by Shanmugaratnam et al. [1] in 1983 and variously reported in literature as teratoid carcinosarcoma, mixed mesodermal tumour, malignant teratoma and blastoma, this rare tumour was aptly termed as "Teratocarcinosarcoma" by Heffner et al. in 1984 [2-4].

This neoplasm is nearly exclusive to the sinonasal tract and arises from pluripotent cells of olfactory epithelium, although rare cases with its primary occurrence in the nasopharynx and oral cavity are reported in literature [2, 58]. Majority of the cases occur in an elderly age group (above 50 years) with a male predominance. However, occasional case reports of its occurrence in children and a single report in neonate are also available $[6,7]$. Majority of the patients present with a short history of nasal obstruction with or without epistaxis and a bone destroying lesion on radiology. Intracranial extension is commonly noted. The radiology clearly indicates the malignant nature but does not spell out the variegated appearance of the lesion.

Histologically, teratocarcinosarcoma is a unique tumour comprising varying combinations of epithelial, mesenchymal and neuroectodermal components exhibiting varying maturation. Epithelial component presents in the form of glands lined by normal appearing columnar to cuboidal and ciliated or nonciliated cells. A focal transition of these glands to squamous epithelium forming morules is noted as was also seen in the present case. Squamous component can be malignant, benign and mature or "fetalappearing" where the cells have clear cytoplasm with distinct cytoplasmic membrane. The presence of these "fetal-appearing" squamous cells is a diagnostic feature as described by Heffner and Hyams [2]. Often, the benign glandular structures are associated with a concentric layer of smooth muscles giving rise to an organoid appearance resembling the primitive bronchial or intestinal organoid structure as seen in teratoma. The organoid pattern of arrangement, irregular shape of the glands, presence of squamous morules or foetal squamous cells and absence of inflammatory response help to distinguish from normal seromucinous glands entrapped in the subepithelial stroma [2]. The poorly differentiated malignant epithelial tissue in the form of sheets may lead to the misdiagnosis of sinonasal undifferentiated carcinoma or squamous carcinoma in a biopsy specimen [9].

The mesenchymal component varies in cellularity and may appear benign to atypical to malignant. Mostly, it is composed of myofibroblastic proliferation with collagenized stroma and less cellular myxoid stroma. Other mature components include cartilage, bone, striated muscles and smooth muscles. These elements may show cytologic atypia and immaturity. Frank malignant sarcomatous transformations such as rhabdomyosarcoma, osteogenic sarcoma or fibrosarcoma, though rare, are also documented [2, 9-11]. However, in the present case, we did not encounter any sarcomatous component.

Undifferentiated round cell component is always present and offers a diagnostic clue [2,9-11]. These undifferentiated round cells may show varying degrees of neuroectodermal and neuroendocrine differentiation mimicking ONB, neuroendocrine carcinoma. These are well delineated by positive labelling with immunohistochemical 
markers such as, synaptophysin, neuron-specific enolase (NSE), chromogranin, NFP and GFAP.

The histomorphology comprising three distinct elements is very specific for the diagnosis of teratocarcinosarcoma [2, 9-11]. This variegated morphology can lead to erroneous interpretation on small biopsies containing only one or two of the three elements. Multiple superficial biopsies may show only benign mesenchymal tissue with scattered benign glands. This should not be dismissed as sinonasal polyp. Many cases could also be diagnosed as poorly differentiated or undifferentiated carcinoma, when malignant epithelial component predominates. Clinicopathologic correlation often indicates mismatch between biopsy findings and imaging findings. History of rapid growth, destructive lesion on radiology and combination of varied components in different biopsies offer a definite clue to the clinician to repeat the biopsy. The first biopsy in our case revealed only round cell component leading to the differential diagnoses of lymphoma, olfactory neuroblastoma and alveolar rhabdomyosarcoma. All of these entities have different therapeutic protocols. The second biopsy revealed the malignant squamous epithelial component, which, if evaluated alone, would have led to the incorrect diagnosis and treatment of a carcinoma. The mesenchymal component seen in the background was mistaken for host stromal response. In the present case, the diagnosis of teratocarcinosarcoma could be rendered only after meticulous evaluation of all the three biopsies together. Young age of the patient was definitely a deterrent point for SNTCS. However, high index of suspicion led to a definitive diagnosis on third biopsy.

The histogenesis of this entity is not yet fully elucidated. Although SNTCS comprise three types of tissue elements or teratoid features, it should not be confused with extragonadal malignant germ cell tumour (GCT) which possesses the potential to develop somatic as well as nonsomatic (extra embryonic) structures. None of the SNTCS, by definition, contain areas of embryonal carcinoma, choriocarcinoma, yolk-sac tumour or seminoma. They have less well-defined organoid or teratoid structures, less developed neuronal tissues and immature/fetal cartilaginous elements as compared with the malignant teratomas. Moreover, all germ cell tumour marker levels (beta-HCG, AFP) are always normal in these tumours. It is hypothesized that this tumour entity arises from primitive pluripotent olfactory epithelial cells, with a capacity to mature in different types of tissues and not from the germ cell as in malignant GCT $[2,10]$.

Teratocarcinosarcoma is a highly malignant neoplasm with extensive local destruction and intracranial extension and requires prompt and aggressive management. The average survival as reported by Heffner and Hyams is
1.7 years [2]. Optimal treatment for SNTCS is still unknown. Combination of radiotherapy and chemotherapy with surgery has been tried with limited success. Prognostic significance of the various histologic components of SNTCS as also the role of chemotherapy has not yet been separately evaluated. In the present case, the patient was treated with a neoadjuvant chemotherapy regimen followed by wide excision. A partial response in the form of reduction in size was noted mainly in the extracranial portion of the tumour as documented on the post-chemotherapy MRI. The predominant component in first and third prechemotherapy biopsies was primitive neuroectodermal tissue lacking maturation. The post-chemotherapy resection specimen was also predominated by neuroectodermal component mainly in the intracranial portion but it exhibited varying degrees of differentiation/maturation in the form of immature ganglion cells in abundant neurofibrillary matrix. This is in contrast to the usual response to the chemotherapy seen in cases of carcinoma. The primitive round cell component was completely missing in this post-neoadjuvant chemotherapy specimen. The morphology of the epithelial components, however, remained unchanged in the post-chemotherapy specimen. This morphologic cellular maturation evidenced by disappearance of the MIC-2 positive undifferentiated/ primitive neuroectodermal component seen in pre-chemotherapy biopsy coupled with the appearance of abundant neurofibrillary matrix in the post-chemotherapy resection specimen is previously not reported in the cases of SNTCS.

It is a well-known phenomenon that morphological maturation of advanced neuroblastoma occurs as a result of cytotoxic anti-tumour chemotherapy with or without maturation-inducing agent, even though the actual mechanism of maturation remains speculative [12]. Post-chemotherapy maturation can also be observed in olfactory neuroblastoma (ONB). Miura $\mathrm{K}$ et al. [13] has reported a bonafide case of ONB which completely transformed into mature ganglioneuroma after chemotherapy. However, maturation can also be seen per primum, though rarely, in ONB which are not treated with neo-adjuvant chemotherapy. Our case exhibited varying degrees of differentiation/maturation in the form of nodules of immature ganglion cells within neurofibrillary matrix. Because the neuronal maturation was localized mainly in the intracranial portion of the tumour in our case the possibility of the role played by tissue milieu i.e., brain parenchyma in inducing maturation needs further evaluation.

Although malignant teratoma and SNTCS are histologically as well as embryologically separate entities, this post-chemotherapy maturation, to some extent, may be comparable to that is seen in malignant teratomas treated with chemotherapy. 
In an unpublished study of 20 cases of SNTCS from our institution, 14 cases had primitive neuroectodermal component. These cases had received various treatment modalities in the form of surgery with or without adjuvant chemotherapy and radiotherapy. However, not even a single case had received neo-adjuvant chemotherapy, as in the present case. To the best of our knowledge, this is the first case in the English literature documenting the morphologic maturation of neuroectodermal elements following administration of chemotherapeutic agents in a case of SNTCS. It remains to be seen if this post-chemotherapy maturation of an undifferentiated, aggressive round cell component to a more differentiated form holds any prognostic significance for better outcome in future prospective studies. Also, this morphologic cellular maturation suggests a very interesting possibility of the potential role of chemotherapeutic agents as differentiation-inducing agents in these highly malignant tumours for which a potentially curative therapeutic regimen has so far been elusive.

To conclude, the tissue heterogeneity in SNTCS often leads to diagnostic errors, emphasizing the need for a high index of suspicion. Neuronal maturation observed in the post-chemotherapy resection specimen holds an early promise for chemotherapeutic agents as differentiationinducing agents that may modify the course of the disease. When the size of mass does not reduce significantly following chemotherapy, a possibility of post-chemotherapy maturation in the neuro-ectodermal component should be kept in mind. Prospective randomized studies are necessary to study the effect of chemotherapy on the other components and to evaluate the prognostic significance of post-chemotherapy maturation in various components. This case is under close observation to evaluate the final outcome.

Open Access This article is distributed under the terms of the Creative Commons Attribution Noncommercial License which permits any noncommercial use, distribution, and reproduction in any medium, provided the original author(s) and source are credited.

\section{References}

1. Shanmugaratnam K, Kunaratnam N, Chia KB, Chiang GS, Sinniah R. Teratoid carcinosarcoma of the paranasal sinuses. Pathology. 1983;15:413-9. doi:10.3109/00313028309085168.

2. Heffner DK, Hyams VJ. Teratocarcinosarcoma (malignant teratoma?) of the nasal cavity and paranasal sinuses. A clinicopathologic study of 20 cases. Cancer. 1984;53:2140-54. doi:10.1002/1097-0142(19840515)53:10<2140::AID-CNCR2820 531025>3.0.CO;2-Y.

3. Patchefsky A, Sundmaker W, Marden PA. Malignant teratoma of the ethmoid sinus: report of a case. Cancer. 1968;21:714-21. doi:10.1002/1097-0142(196804)21:4<714::AID-CNCR28202104 24>3.0.CO;2-J.

4. Petrovich Z, Wollman J, Acquarelli M, Barton R. Malignant teratoma of nasal cavity. J Surg Oncol. 1977;9:21-8. doi:10.1002/ jso. 2930090105.

5. Meinecke R, Bauer F, Skouras J, et al. Blastomatous tumours of the respiratory tract. Cancer. 1976;38:818-23. doi:10.1002/10970142(197608)38:2<818::AID-CNCR2820380225>3.0.CO;2-1.

6. Rotenberg B, El-Hakim H, Lodha A, et al. Nasopharyngeal teratocarcinosarcoma. Int J Pediatr Otorhinolaryngol. 2002;62: 159-64. doi:10.1016/S0165-5876(01)00575-4.

7. Crazzolara R, Puelacher W, Ninkovic M, et al. Teratocarcinosarcoma of the oral cavity. Pediatr Blood Cancer. 2004;43: 687-91. doi:10.1002/pbc.20139.

8. Carrizo F, Pineda-Daboin K, Neto AG, Luna MA. Pharyngeal teratocarcinosarcoma: review of the literature and report of two cases. Ann Diagn Pathol. 2006;10:339-42. doi:10.1016/j.anndiag path.2006.03.006.

9. Pai SA, Naresh KN, Masih K, Ramarao C, Borges AM, Path FRC. Teratocarcinosarcoma of the paranasal sinuses: a clinicopathologic and immunohistochemical study. Hum Pathol. 1998; 29:718-22. doi:10.1016/S0046-8177(98)90281-7.

10. Wei S, Carroll W, Lazenby A, Bell W, Lopez R, Said-Al-Naief N. Sinonasal teratocarcinosarcoma: report of a case with review of literature and treatment outcome. Annal Diagn Pathol 2007. www.sciencedirect.com. Accessed 08 October 2007.

11. Shemen L, Galantich P, Murali R. Malignant teratocarcinosarcoma of sphenoid sinus. Otolaryngol Head Neck Surg. 1995;112: 496-500. doi:10.1016/S0194-5998(95)70294-6.

12. Ogita S, Tokiwa K, Arizono N, Takahashi T. Neuroblastoma: incomplete differentiated on the way to maturation or morphological alteration resembling maturity? Oncology. 1988;45: 148-52.

13. Miura K, Mineta H, Yokota N, Tsutsui Y. Olfactory neuroblastoma with epithelial and endocrine differentiation transformed into ganglioneuroma after chemoradiotherapy. Pathol Int. 2001;5: 942-7. 